\section{Squaring the RNA circle}

\section{By Chris Cain, Senior Writer}

Two European teams have provided the strongest evidence to date that circular RNAs are widely expressed in human cells and have clear regulatory functions. ${ }^{1,2}$ The biomedical relevance of circular RNAs remains largely unexplored, but the recent explosion of studies linking noncoding RNAs to disease may portend a similar trajectory for this emerging class of nucleic acids.

The refinement of high throughput sequencing methods over the past decade has led to the discovery of thousands of noncoding RNAs, including numerous microRNAs and long noncoding RNAs. Although initial studies were primarily catalogs of RNA transcripts, ${ }^{3}$ subsequent functional analyses demonstrated that these molecules have widespread regulatory roles in human diseases. ${ }^{4}$

Numerous companies are now developing therapeutics or diagnostics that use or target miRNAs or lncRNAs in indications ranging from cardiovascular disease to cancer.

Although a handful of studies had suggested some individual RNA transcripts might exist in a circular form, until last year there were no reports of widespread RNA circles. That changed in 2012, when separate groups from Stanford University and The University of North Carolina at Chapel Hill School of Medicine published analyses of RNA sequencing data from human cells, suggesting thousands of transcripts may exist in a circular form. ${ }^{5,6}$

Norman Sharpless, who led one of the studies and is professor of medicine and genetics at the UNC School of Medicine, told SciBX that the discovery of abundant RNA circles was unexpected.

"At the time we started our study, there were maybe a handful known in mammals, and people thought of them as rare, low-copy artifacts that could be due to the occasional RNA splicing malfunction. But we found thousands of them-they are in some cases expressed at levels higher than the linear form of the transcript, and they are conserved in mouse and humans," he said.

Despite the striking results, neither study provided evidence of a specific molecular function for circular RNAs.

Now, independent teams at the Max Delbruck Center for Molecular Medicine and Aarhus University have shown that circular RNAs have clear functional significance.
Both teams focused on the same circular RNA, cerebellar degeneration-related protein 1 antisense (ciRS-7; CDR1as), because computational analysis predicted more than 70 miRNA-7 (miR-7) binding sites within the transcript. They speculated that $c i R S-7$ could act as a stable RNA sponge that could sequester miR-7 and prevent it from acting on its downstream targets.

To test this, the teams used immunoprecipitation experiments to show that miR-7 directly bound ciRS-7. Subsequent functional experiments showed that knockdown of $c i R S-7$ increased expression of miR-7 target genes including epidermal growth factor receptor (EGFR) and $\alpha$-synuclein (SNCA) compared with no knockdown.

Neither team could detect a linear form of $c i R S-7$ in cells, whereas the circular form was abundant and highly stable.

In mice, the teams used immunohistochemistry to show that $c i R S-7$ and miR-7 are coexpressed in brain tissue. The Max Delbruck team further showed that in zebrafish, injecting ciRS-7 or knocking down miR-7 caused similar defects in brain development.

Finally, to expand the relevance of the results beyond $c i R S-7$, the Max Delbruck team used a computational approach to identify about 2,000 additional circular RNAs in a human cell line. The group chose a subset of transcripts to experimentally validate and confirmed 19 of 23 (83\%). The Aarhus team did not take a systematic approach but instead showed that another circular RNA, sex-determining region $Y(S R Y)$ locus, contained binding sites for miR138 and had a similar miRNA sequestration function.

Both studies were published in Nature.

-Nikolaus Rajewsky, Medicine

\section{Circular functions}

More extensive profiling of circular RNAs is needed before the extent of their biomedical relevance becomes clear. Nevertheless, there are parallels between the discovery of circular RNAs and other noncoding RNAs that recently have become appreciated as drug targets.

Nikolaus Rajewsky, professor of systems biology and leader of the research team at Max Delbruck, told SciBX that RNA circles are just now being identified because of prior conceptual and technical limitations.

"Throughout the RNA world, one lesson has been that whenever you look for something that would not have been seen for technical reasons, you end up finding something new," he said. "When people found small RNAs, it was thought to be degradation for a long time, but then it suddenly turned out it was tremendously important. Now we have found RNAs that are covalently closed and are functionally important."

Julia Salzman, a postdoc at Stanford University who published the first catalog of widespread RNA circle expression early last year, ${ }^{5}$ agreed that researchers' mindsets have been a limiting factor in identifying circular RNA. "One difficulty with their identification has been that most people ignore sequencing reads that aren't consistent with the current models of how splicing takes place," she said.

She added that a technical difficulty with identifying circular RNA 
has been that most sequencing protocols use methods to select for polyadenylated (polyA) transcripts. Those methods would miss circular RNAs, which lack a polyA tail.

"I think the vast majority of available data is based on polyA-selected samples, and that makes a comprehensive study of the phenomenon more difficult," she said.

She added that the progress made in identifying circular RNAs over the last year has opened up an entire field of new molecules for functional exploration. "These papers are promising and exciting. RNA circles are so abundant and so diverse that it will lead to fruitful research efforts for many groups for many years," she said.

Salzman said it was important to note that the papers focused primarily on a noncoding RNA found only in a circular form. Many RNAs exist in both circular and linear forms, and thus their functions will be much harder to tease apart, she said.

Salzman will continue her circular RNA studies in her own lab as an assistant professor at Harvard Medical School in the fall.

Arthur Krieg, president, cofounder and CEO of RaNA Therapeutics Inc., agreed that the results will spur intense follow-up. "The way the RNA field has been going, this will generate significant interest, and people will be jumping on these results in the coming months," he said. "I think from the therapeutic perspective, this could be yet another category of targets for us to pursue, and we now have the benefit of 20 years of advances in developing oligonucleotide therapeutics to guide us."

RaNA is developing oligonucleotides that block the interaction between $\operatorname{lncRNAs}$ and polycomb repressive complex 2 (PRC2) to activate the expression of specific genes.

Sharpless cited lncRNAs as an example of how rapidly new classes of RNA have been linked to disease. First identified in abundance in 2005 , lncRNAs are now associated with many human diseases, and in the last six months, new lncRNAs have been characterized with roles in inherited human diseases and in regulating the immune response.,8

"This is an emerging field, and while I am not sure what the clinical and commercial implications of RNA circles are, I am sure these will turn out to be really important in some way that is not readily apparent," he said.

Sharpless thinks circular RNA acting as a miRNA sponge may be only one example of how it can function. Sharpless is continuing to study the regulation of the cyclin dependent kinase inhibitor $2 A$ (CDKN2A; INK4a; $A R F ; p 16 I N K 4 a$ ) and CDKN2B (INK4B; MTS2) locus by the lncRNA CDKN2B antisense RNA 1 (CDKN2B-AS1; ANRIL) and its recently identified circular isoform, $c A N R I L$.

Rajewsky also thinks the functions of circular RNAs likely extend beyond miRNA binding. "There is no reason to think that miRNAs are the only molecule they interact with, and we are systematically fishing for other molecules that sit on circular RNAs," he said.

Pier Paolo Pandolfi, professor of medicine and pathology at Harvard Medical School and director of research at the Cancer Center at Beth Israel Deaconess Medical Center, agreed that circular RNAs as well as other noncoding RNAs may have diverse functions, including their ability to act as competitive endogenous RNAs (ceRNAs) for miRNAs. He said the identification of circular RNAs as miRNA sponges provides an additional piece of evidence that competition for miRNA binding plays a key regulatory role within cells and in disease pathogenesis.

Recently, his group has been one of the leading proponents of an emerging hypothesis that competition between transcripts for miRNA binding has functional consequences, and he has demonstrated a regulatory role for ceRNAs in controlling the tumor suppressor PTEN (MMAC1; TEP1). ${ }^{9-11}$

\section{Circling drug development}

Although the jury is still out on the extent of circular RNA's role in disease, MiReven Pty. Ltd. thinks the specific circular RNA identified by these studies could have direct implications for its oncology program.

MiReven was founded in 2010 to develop derivatives of miR-7 to treat cancer. Work published by its academic founders showed that miR-7 directly represses the expression of known oncogenes including EGFR. The program is in preclinical development.

Christopher Wraight, technology advisor and manager for MiReven, told SciBX that he wanted to see more details about where $c i R S-7$ is expressed. "Our aim is to increase expression and activity of miR-7 in tumors, so clearly the reported observations that destruction of ciRS-7 released increased levels of functional miR-7 into the cell is relevant. The tissue distribution of these RNAs is important, and it would appear that the coexpression of

-Arthur Krieg, miR-7 and $c i R S-7$ in the brain mak
treatment of CNS tumors," he said.

He added that in situations in which it might be desirable to knock down miRNA expression, circular RNAs could be engineered as an alternative to current antagomir approaches.

Indeed, Jørgen Kjems, professor of molecular biology at Aarhus University and corresponding author of one of the papers, said his team now plans to knock down $c i R S-7$ and develop circular RNAs as potential therapeutics.

"Although we have only proven it for miR-7, a circular RNA should work on any other microRNA if the sequence is redesigned, and this is in fact something we are currently investigating," Kjems said.

In addition, the Aarhus team showed that another miRNA, miR671, cleaves $c i R S-7$, and now the team is considering testing miR-671 in models for cancer and Parkinson's disease (PD).

Rajewsky's next steps include analyzing the expression of circular RNAs in patient samples.

"We are running a project on looking at circular RNA expression in disease. Because they are stable and we have shown they are tissue specific, they could be good biomarkers. A good biomarker is of course difficult-there have been lots of promises and not too much success, but this is a completely novel class of molecules to look for, and we plan to screen, for example, blood, urine and saliva for circular RNAs," he said.

Both teams intend to continue functional studies to develop a better molecular understanding of the specific factors required for RNA 


\section{ANALYSIS}

circularization.

Results from Kjems are patented and available for licensing from Aarhus University. The patent and licensing status of the findings from the Max Delbruck team is undisclosed.

Cain, C. SciBX 6(10); doi:10.1038/scibx.2013.229

Published online March 14, 2013

\section{REFERENCES}

1. Memczak, S. et al. Nature; published online Feb. 27, 2013; doi:10.1038/nature11928

Contact: Nikolaus Rajewsky, Max Delbruck Center for Molecular Medicine, Berlin, Germany e-mail: rajewsky@mdc-berlin.de

2. Hansen, T.B. et al. Nature; published online Feb. 27, 2013; doi:10.1038/nature11993

Contact: Jørgen Kjems, Aarhus University, Aarhus, Denmark e-mail: jk@mb.au.dk

Contact: Thomas B. Hansen, same affiliation as above e-mail: tbh@mb.au.dk
3. Kapranov, P. et al. Science 316, 1484-1488 (2007)

4. Esteller, M. Nat. Rev. Genet. 12, 861-874 (2011)

5. Salzman, J. et al. PLoS ONE 7, e30733; published online Feb. 1, 2012; doi:10.1371/journal.pone.0030733

6. Jeck, W.R. et al. RNA 19, 141-157 (2013)

7. Troy, A. \& Sharpless, N.E. J. Clin. Invest. 122, 3837-3840 (2012)

8. Gomez, J.A. et al. Cell 152, 743-754 (2013)

9. Salmena, L. et al. Cell 146, 353-358 (2011)

10. Tay, Y. et al. Cell 147, 344-357 (2011)

11. Karreth, F.A. et al. Cell 147, 382-395 (2011)

COMPANIES AND INSTITUTIONS MENTIONED

Aarhus University, Aarhus, Denmark

Beth Israel Deaconess Medical Center, Boston, Mass.

Harvard Medical School, Boston, Mass.

Max Delbruck Center for Molecular Medicine, Berlin, Germany MiReven Pty. Ltd., Perth, Western Australia, Australia

RaNA Therapeutics Inc., Cambridge, Mass.

Stanford University, Stanford, Calif.

The University of North Carolina at Chapel Hill School of Medicine, Chapel Hill, N.C. 